\title{
Effet des facteurs de l'environnement sur les Fusarium pathogènes des plantes cultivées
}

\author{
Jacques S. B. DOSSA ${ }^{1 *}$, Euloge C. TOGBE ${ }^{2}$, Michaël PERNACI ${ }^{3}$, \\ Euloge K. AGBOSSOU ${ }^{2}$ et Bonaventure C. AHOHUENDO ${ }^{2}$
${ }^{1}$ Centre de Recherches Agricoles Plantes Pérennes, Institut National des Recherches Agricoles du Bénin, BP: 01 Pobè, Benin.
${ }^{2}$ Faculté des Sciences Agronomiques, Université d'Abomey-Calavi BP: 526 FSA / UAC Cotonou Benin.
${ }^{3}$ PalmElit SAS, Agropolis Park, Bat 14, 2214 Bd de La Lironde, F-34980, Montferrier sur Lez, France.
*Auteur correspondant ; E-mail : dossajacques@yahoo.fr; Tel : (+229) 97643084

\section{RESUME}

Les Fusarium pathogènes des plantes cultivées dont Fusarium oxysporum f. sp. elaeidis agent de la fusariose du palmier à huile, sont des espèces de champignons phytopathogènes rencontrées dans les sols cultivés aussi bien des régions tempérées que des régions tropicales. Ils s'attaquent aux cultures et engendrent des dégâts économiques conséquents. Cette synthèse fait le point des études menées sur les effets des facteurs de l'environnement sur le développement et le potentiel infectieux des espèces pathogènes du genre Fusarium. Les variables climatiques à savoir les précipitations, l'humidité du sol et de l'air, l'ensoleillement et la température sont autant de facteurs impactant le développement des Fusarium. La salinité de l'air et du sol agit de façon directe ou indirecte sur leur biologie et leur cycle de vie. A forte concentration, le chlorure de sodium stimule certaines espèces de Fusarium alors qu'il entraine une forte réduction de la densité d'autres espèces. De plus, la composition chimique, la communauté microbienne ainsi que le $\mathrm{pH}$ du sol modulent également leur développement. Enfin les éléments minéraux tels que le Fer, le Manganèse, le Zinc, le Calcium, le Potassium, le Sodium et le Magnésium peuvent induire des mécanismes de résistance chez les plantes en réponse aux attaques causées par les Fusarium. Cependant, il faut noter que les effets des facteurs climatiques peuvent être bénéfiques ou non en fonction des espèces. Il en est de même pour le pH, les communautés microbiennes, les éléments minéraux et les sels notamment le $\mathrm{NaCl}$ contenus dans les sols dont nous constatons que les effets diffèrent d'une espèce à l'autre. Ces résultats ne sont donc pas extrapolables au couple Fusarium oxysporum f. sp. elaeidis - palmier à huile. Par conséquent, la collecte des données pouvant permettre l'identification des différents facteurs agissant sur Fusarium oxysporum f. sp. elaeidis devient pertinente. De plus, ces connaissances contribueraient à optimiser les tests de screening pour la sélection du matériel tolérant et permettraient une meilleure gestion intégrée de la fusariose en plantation.

(C) 2019 International Formulae Group. All rights reserved.

Mots clés : Développement Fusarium, facteurs climatiques, composition sol, pH, salinité.

\section{Effect of environmental factors on pathogenic Fusarium in crops}




\section{ABSTRACT}

Fusarium pathogens from cultivated plants, including Fusarium oxysporum f. sp. elaeidis, agent of Fusarium wilt of oil palm, are species of phytopathogenic fungi found both in cultivated soils in temperate and tropical regions. They attack crops and cause significant economic damage. This review summarizes studies conducted on the effects of environmental factors on the development and infectious potential of these pathogenic Fusarium. Climate variables such as precipitation, soil and air humidity, sunlight and temperature are all factors that impact the development of Fusarium. The salinity of the air and soil acts directly or indirectly on their biology and life cycle. In high rate concentration, sodium chloride stimulates some Fusarium species whereas it causes a strong reduction in the density of other species. In addition, the chemical composition, microbial community and soil $\mathrm{pH}$ also modulate their development. Finally, mineral elements such as iron, manganese, zinc, calcium, potassium, sodium, and magnesium can induce resistance mechanisms in plants in response to attacks caused by Fusarium. This review shows that climatic factors act on the development of Fusarium. However, it should be noted that the effects of climatic factors may or may not be beneficial regarding the species. The same is true for $\mathrm{pH}$, microbial communities, mineral elements and salts, mainly $\mathrm{NaCl}$ contained in soils, whose effects differ from one species to another. These results cannot therefore be extrapolated to Fusarium oxysporum f. sp. elaeidis - oil palm. Therefore, the collection of data that may allow the identification of the different factors acting on Fusarium oxysporum f. sp. elaeidis becomes relevant. Moreover, this knowledge would help to optimize screening tests for the selection of tolerant material and would allow better integrated management of fusariosis in plantations.

(C) 2019 International Formulae Group. All rights reserved.

Keywords: Fusarium development, climatic factors, soil composition, pH, salinity.

\section{INTRODUCTION}

Les espèces du genre Fusarium (Fusarium sp) sont communément associées aux végétaux supérieurs et font partis des champignons les plus rencontrés dans les écosystèmes terrestres (Ploetz, 2001) et les plus fréquemment isolés par les phytopathologistes. Le genre Fusarium comprend certaines des espèces de champignons phytopathogènes d'importance économique les plus grandes pour l'agriculture et l'horticulture (Summerell et al., 2010 ; Ma et al., 2013; Gordon, 2017). La diversité des hôtes infectés par Fusarium sp, le nombre de taxa pathogènes et les types d'habitats dans lesquels ils sont présents en font un cas particulier en phytopathologie (Leslie et al., 2006). Ainsi, les Fusarium phytopathogènes sont rencontrés aussi bien dans les sols cultivés des régions tempérées que des régions tropicales. Dans les régions tempérées, de nombreuses cultures sont attaquées par les Fusarium engendrant des dégâts économiques conséquents. Ainsi Fusarium graminearum et Fusarium culmorum sur le blé (Xu et Nicholson, 2009 ; Walter et al., 2010), Fusarium solani var. coeruleum sur la pomme de terre (Mecteau et al., 2008), Fusarium oxysporum f. sp. lactucae sur la laitue (Scott et al., 2010; Ferrocino et al., 2013), Fusarium graminearum sur le maïs (Scauflaire et al., 2011) et Fusarium oxysporum f. sp. lycopersici sur la tomate (Gnancadja et al., 2015) font subir de lourdes pertes à ces cultures. A titre d'exemple, les Fusarium ont occasionnés 2,7 milliards de dollars de perte en production de blé dans les états du Nord et du Centre des Etats-Unis entre 1998 et 2002 (Nganje et al., 2002). En zone tropicale, les maladies causées par des espèces du genre Fusarium sont également nombreuses et provoquent des pertes conséquentes sur des cultures qui revêtent un rôle économique central. Il s'agit de Fusarium oxysporum f. sp. cubense sur le bananier (Ploetz, 2006 ; Kra et al., 2011), Fusarium xylarioides sur le caféier (Geiser et al., 2005 ; Rutherford, 2006), Fusarium oxysporum f. sp. vasinfectum sur le cotonnier (Bennett et al., 2011), Fusarium subglutinans sur l'ananas (Borràs et al., 2001), Fusarium verticillioides sur la canne à sucre (Abbas et al., 2012) et Fusarium moniliforme sur le riz (Zehhar et al., 2006 ; Shahadat Hossain et al., 2015). Par 
ailleurs en Afrique, la fusariose vasculaire du palmier à huile provoquée par Fusarium oxysporum f. sp elaeidis est la principale maladie qui affecte cette grande culture (Flood, 2006; de Franqueville et al., 2011; Diabaté et al., 2013; Gogbe et al., 2016), causant jusqu'à $70 \%$ de mortalité en plantation.

Des études ont montré que la sévérité des maladies causées par les Fusarium dépend des facteurs environnementaux ( $\mathrm{Xu}$ et al., 2008b ; Ferrigo et al., 2016) et climatiques (Rossi et al., 2001; Dohan et al., 2003). La compréhension de l'effet de ces différents facteurs sur le développement et l'incidence des Fusarium sp pourrait fournir des informations précieuses pour la compréhension de la biologie des Fusarium, le développement de méthodes de lutte intégrées, la compréhension des mécanismes épidémiologiques et l'élaboration ou le perfectionnement de tests de résistance variétale. La présente revue fait le point des résultats des recherche de l'influence du climat (à travers les saisons), des sels (notamment le chlorure de sodium) et les statuts chimiques et biologiques du sol sur le développement, la sévérité et la propagation des fusarioses afin d'identifier les facteurs impactant potentiellement leur expression. Ceci permettrait d'ouvrir des pistes d'investigations pour le Fusarium oxysporum f. sp elaeidis, responsable de la fusariose du palmier à huile, en vue de l'amélioration du processus de sélection de palmier tolérant et de développement de méthodes de gestion intégrées de cette maladie en plantation.

\section{EFFET DES FACTEURS CLIMATIQUES SUR LE DEVELOPPEMENT DES FUSARIUM}

Les facteurs climatiques, en particulier l'humidité et la température, jouent un rôle primordial dans le développement des Fusarium en conditionnant la germination et l'infection de ceux-ci (Siou, 2013). Selon Bérubé et al. (2009), le climat est le facteur le plus déterminant dans le développement des Fusarium. Ces auteurs ont identifié la pluie et l'humidité relative de l'air comme deux variables climatiques favorisant l'expansion de la fusariose de l'épi du blé et de l'orge. En effet, la pluie et le vent constituent les moyens de transport des spores produites par $F$. graminearum vers les épis ou les soies (Alvarez et al., 2010) et l'infection par ce Fusarium survient pendant la période épiaison-floraison chez les céréales et la sortie des soies chez le maïs (Bailey et al., 2004). De plus selon ces auteurs, la croissance du Fusarium se poursuit aussi longtemps que les conditions d'humidité le permettent.

La température influence le développement des Fusarium, et constitue de manière générale un paramètre essentiel du développement des champignons phytopathogènes (Bernard, 2012). Elle influence fortement la plupart des processus métaboliques des organismes vivants, et affecte presque tous les aspects de leur croissance et de leur développement. Il a été notamment montré qu'elle influence fortement la production de spores chez les Fusarium (Doohan et al., 2003). Chaque espèce de Fusarium a un optimum de température et d'humidité pour laquelle l'expression de la maladie est maximale. Ainsi $F$. graminearum et $F$. avenaceum ont leur optimum à $28-29^{\circ} \mathrm{C}$ alors que $F$. culmorum a son optimum à $26,5^{\circ} \mathrm{C}$ (Rossi et al., 2001). Il faut néanmoins souligner que des individus de la même espèce mais ayant des origines géographiques différentes vont également avoir des optima différents en lien avec le climat de leur région d'origine (Doohan et al., 2003; Xu et Nicholson, 2009). Les facteurs météorologiques tels que la pluie, la rosée, le brouillard, la vitesse du vent, l'ensoleillement, la température et l'humidité relative agissent sur les différentes phases de développement des Fusarium. En conditions de température, d'humidité relative et avec un ensoleillement favorable, les spores des Fusarium peuvent survivre en absence d'hôte (Awad et al., 2010). Les fluctuations saisonnières de température et d'humidité du sol, observées sur les populations de F. culmorum dans le sol, ont un effet sur la sévérité de la fusariose 
du blé (Bateman et al., 2001). En effet, ces auteurs ont montré qu'à la fin de l'automne et au début de l'hiver, du fait de l'augmentation de l'humidité relative et de la diminution de la température, la densité de $F$. culmorum diminuait dans le sol, et inversement. Un effet identique de la température sur les densités de spores de $F$. oxysporum f. sp. lactucae dans le sol a été reporté, qui se traduit par une sévérité plus élevée de la fusariose de la laitue lorsque la température augmente (Scott et Gordon, 2010; Ferrocino et al., 2013). L'étude de l'effet combiné de la température et de l'humidité sur deux souches de $F$. graminearum a montré que la croissance mycélienne atteint son niveau optimum à 25 ${ }^{\circ} \mathrm{C}$ avec une activité de l'eau (i.e quantité d'eau libre nécessaire au développement des microorganismes) comprise entre 0,950 et 0,995 (Ramirez et al., 2006).

\section{EFFETS DE LA COMPOSITION DU SOL SUR LE DEVELOPPEMENT DES FUSARIUM}

Les activités biologiques et les propriétés physico-chimiques du sol présentent une forte interdépendance (Boudoudou et al., 2009). En effet, les études ont montré que les éléments chimiques favorisent ou inhibent la croissance, la germination et la sporulation des Fusarium (Nyiransengiyumva, 2007). Les sols argileux à forte capacité d'échange cationique et riche en éléments nutritifs favorisent l'installation et le développement des Fusarium et les sols ayant un pourcentage élevé en sable et une sodicité supérieure à $30 \%$ présentent une faible charge fongique (Boudoudou et al., 2009). Les sols pauvres en matières organiques ont une faible capacité d'accueil des Fusarium (Mahdi, 2011). La fertilisation organique conduit à l'augmentation de la teneur en carbone organique dans le sol (Mohamed, 2006), ce qui favorise le développement des microorganismes antagonistes aux Fusarium (Alabouvette et al., 2009).

Certains éléments minéraux du sol agissent aussi sur le développement des
Fusarium. C'est ainsi que les composés calciques inorganiques tels que le silicate, l'hydroxyde et l'oxyde de calcium sont très efficaces dans l'inhibition de la croissance mycélienne, la production des conidies et la germination de $F$. oxysporum et $F$. avenaceum (Attrassi et Rahouti, 2016). Au contraire, le phosphore favorise le développement de certaines espèces de Fusarium. C'est ce qui explique que des flétrissements fusariens importants ont été observés suite à des apports de composés phosphatés sur la tomate cultivée en pots et au champ, sur le coton et le cantaloup dont les agents responsables sont respectivement $F$. oxysporum f. $\mathrm{sp}$. Lycopersici, $F$. oxysporum f. sp. vasinfectum et $F$. oxysporum f. sp. melonis (Duffy et Défago, 1999; Lambert et al., 2005).

Le potassium inhibe également le développement de certains Fusarium. Ainsi une réduction moyenne de $36 \%$ de la sévérité de la mort subite du soja causé par $F$. solani f. sp. glycines a été observée suite à l'application du chlorure de potassium comparativement au traitement témoin (Sanogo et Yang, 2001). Par ailleurs, la fumure potassique a un effet suppressif à long terme sur la fusariose du palmier à huile causée par Fusarium oxysporum f. sp. elaeidis (Ollagnier et Renard, 1976).

Le chlorure de magnésium peut également favoriser ou inhiber le développement des Fusarium. Ainsi le chlorure de magnésium n'affecte pas la croissance de $F$. oxysporum f. sp. radicislycopersici (Duffy et Défago, 1999), alors qu'il a été observé une augmentation du flétrissement fusarien causé par $F$. oxysporum f. sp. lycopersici chez la tomate et par $F$. oxysporum f. sp. apii chez le céleri, suite à l'apport du chlorure de magnésium (Lambert et al., 2005).

Les teneurs élevées du sol en oligoéléments tels que le fer, le zinc et le cuivre ralentissent le développement des Fusarium. Ceci se traduit par une diminution des infections que ces champignons engendrent (Martinez et al., 2002), particulièrement le zinc qui induit une diminution de la croissance 
mycélienne et du pouvoir pathogène de $F$. culmorum (Djébali et al., 2014). L'équilibre des éléments minéraux dans le sol favorise leur utilisation par les plantes pour leur croissance et leur résistance contre les Fusarium (Wopereis et al., 2008). Ainsi, il a été montré que des teneurs élevées en sodium accompagnées de faibles teneurs en calcium et en magnésium diminuent la résistance des bananiers à l'attaque du $F$. oxysporum var. cubense (Dominguez et al., 2003). Par ailleurs, du fait du rôle primordial du magnésium dans la photosynthèse, sa déficience se manifeste par un affaiblissement général rendant ainsi la plante vulnérable aux infections fongiques (Mohamed, 2006).

\section{EFFETS DU PH SUR LE DEVELOPPEMENT DES FUSARIUM}

Le $\mathrm{pH}$ du sol affecte également le développement des Fusarium, car la croissance mycélienne et la germination des conidies sont restreintes à une certaine gamme pour chaque espèce. Cette gamme de $\mathrm{pH}$ peut être large ou restreinte en fonction des espèces. Ainsi $F$. graminearum et $F$. culmorum (Beyer et al., 2004) et Fusarium oxysporum f. sp. glycines (Balasu et al., 2015) peuvent crôtre sur des milieux dont le $\mathrm{pH}$ varie respectivement entre 4 et 10 et entre 4 et 7. Par ailleurs, les sols ayant un $\mathrm{pH}$ compris entre 5 et 7 sont favorables à la survie et à la germination des chlamydospores, ainsi qu'à la croissance mycélienne de Fusarium oxysporum f. sp. elaeidis (Oritsejafor, 1986a). Par contre cette gamme de $\mathrm{pH}$ est réduite pour d'autres espèces de Fusarium. Ainsi, le pH optimal de croissance de Fusarium moliniforme est de 6 (Shahadat Hossain et al., 2015) alors que le $\mathrm{pH}$ optimal de croissance et de sporulation de Fusarium udum (Chaudhary et al., 2018) et Fusarium oxysporum f. sp. ciceri (Khilare et Ahmed, 2012) est compris entre 6 et 6,5 .

\section{EFFETS DE LA SALINITE SUR LE DEVELOPPEMENT DES FUSARIUM}

La salinité du sol et de l'air constitue l'un des éléments de l'environnement qui conditionnent la survie, le développement et le degré d'infection des champignons phytopathogènes (Regragui, 2005). L'action des sels sur les Fusarium peut être favorable ou défavorable et varie d'une espèce à l'autre. Ainsi le chlorure d'ammonium réduit la croissance et le développement de Fusarium solani var coeruleum (Mecteau et al., 2008). Les sels de calcium inhibent les trois stades du cycle de vie de $F$. oxysporum, responsable de la pourriture du melon en post-récolte (Zemmouri et al., 2015). Le $\mathrm{NaCl}$ peut inhiber ou favoriser le développement des Fusarium. Ainsi il a été constaté que le $\mathrm{NaCl}$ réduit le développement de Fusarium oxysporum f. sp. asparagi et Fusarium prolifératum (Reid et al., 2001). Par contre Keren (2000) a observé une augmentation significative de la croissance du mycélium suite à une augmentation de la concentration en $\mathrm{NaCl}$ du milieu sur Fusarium oxysporum albendinis. Daami Remadi et al. (2009) ont observé que le $\mathrm{NaCl}$ n'a pas d'effet significatif sur la croissance mycélienne de Fusarium oxysporum f. sp. lycopersici mais une sporulation accrue est observée pour des concentrations de $\mathrm{NaCl}$ élevées.

\section{RECEPTIVITE DES SOLS AUX FUSARIUM}

Les facteurs édaphiques et biotiques du sol ainsi que la disponibilité en éléments minéraux peuvent conditionner l'activité des Fusarium pathogènes. Toutefois, ces différents facteurs n'agissent pas indépendamment mais peuvent se moduler. Ces interactions entre facteurs abiotiques et biotiques du sol en lien avec le développement des Fusarium pathogènes ont été conceptualisées par Louvet et al. (1976), conduisant à la notion de réceptivité des sols. La réceptivité du sol est la résultante des facteurs de l'environnement biotique et abiotique du sol qui conditionne le maintien et le développement de l'agent pathogène. Ainsi dans le cas de la fusariose du bananier, il a été montré que les interactions entre agents microbiens sont responsables du caractère suppressif des sols de bananeraies vis-à-vis de 
F. oxysporum f. sp. cubense (Alabouvette et al., 2001). Ces interactions biotiques dépendent des caractéristiques abiotiques du sol, notamment du $\mathrm{pH}$ et de sa nature argileuse. De plus, la concurrence pour les nutriments, principalement le carbone et le fer, est l'un des mécanismes qui détermine le caractère suppressif naturel de ces sols aux fusarioses vasculaires (Alabouvette 1999).

\section{Conclusion}

Cette revue montre que les facteurs climatiques agissent sur le développement des Fusarium. Cependant il faut noter que leurs effets peuvent être bénéfiques ou non en fonction des espèces. Il en est de même pour le $\mathrm{pH}$, les communautés microbiennes, les éléments minéraux et les sels notamment le $\mathrm{NaCl}$ contenus dans les sols dont nous constatons que les effets diffèrent d'une espèce à l'autre. Cette situation rend problématique la généralisation de ces effets à Fusarium oxysporum f. sp. elaeidis. Par conséquent, les études des effets de ces différents facteurs sur le couple Fusarium oxysporum f. sp. elaeidis - palmier à huile s'imposent pour l'identification des facteurs agissant sur ce champignon et qui peuvent potentiellement impacter l'expression de la fusariose. L'identification des facteurs impactant son développement et son expression permettrait d'optimiser la conduite des tests de screening visant à sélectionner du matériel tolérant à la fusariose. De plus, ces données pourraient être utiles à la gestion de la fusariose en plantation et contribuer à une gestion intégrée de cette maladie, notamment lors de la mise en place de nouvelles plantations.

\section{CONFLIT D'INTERETS}

Les auteurs déclarent n'avoir aucun conflit d'intérêts

\section{CONTRIBUTIONS DES AUTEURS}

SBJD, CET et MP ont collecté les informations et rédigé le manuscrit. KEA et CBA ont suppervisé la collecte des informations, lu et corrigé le manuscrit.

\section{REFERENCES}

Abbas M, Reza Farrokhi N, Nasrin Noras M. 2012. Fusarium verticillioides from surgarcane, vegetative compatibility group and pathogenicity. Plant Protection Science, 48(2): 80-84.

Alabouvette C. 1999. Fusarium wilt suppressive: an example of diseasesuppressive soils. Australasian Plant Pathology, 28: 57-64.

Alabouvette C, Edel V, Lemeceau P, Olivain C, Recorbet G, Steinberg C. 2001. Diversity and interactions among stain of Fusarium oxysporum: application to biological control. In, Biotic Interactions in Plant Pathogen Association. Jeger MJ, Spence NJ. (ed) Wallingford, CAB International; 131-158.

Alabouvette C, Olivain Q, Migheli C, Steinberg C. 2009. Microbiological control of soil-borne phytopathogenic fungi with special emphasis on wiltinducing Fusarium oxysporum. New Phytologist., 184(3): 529-544. DOI: 10.1111/j.1469-8137.2009.03014.x.

Alvarez C L, Somma S, Moretti A, Fernando Pinto V. 2010. Agressiveness of Fusarium graminearum sensu stricto isolates in wheat kernels in Agentina. Journal of Phytopathology, 158: 173181. DOI: https://doi.org/10.1111/j.14390434.2009.01596.x

Attrassi K, Rahouti M. 2016. Effet de composés calciques inorganiques sur le développement in vitro de moisissures isolées d'agrumes après la récolte. Bulletin de la Société Royale des Sciences de Liège, 8: 263-275.

Awad W A, Ghareeb K, Bohm J, Zentek J. 2010. Decontamination and detoxification strategies for the Fusarium mycotoxin deoxynivalenol in animal feed and the effectiveness of microbial biodegradation. Food Addit. Contam. Part. A-Chem., 27(4): 510-520. DOI:10.1080/19440040903571747.

Bailey KL, Couture L, Gossen BD, Gugel RK, Morrall RAA. 2004. Maladies des 
grandes cultures au Canada. Éd. La Société canadienne de Phytopathologie.

Balasu AG, Cristea S, Zala CR, Oprea M. 2015. The biological growth parameters of the Fusarium oxysporum f. sp. glycines fungus. Romanian Biotechnological Letters, 20(6): 1092110928 .

Bateman GL, Murray G. 2001. Seasonal variations in populations of Fusarium species in wheat-field soil. Applied Soil Ecology, 18: 117-128. DOI: https://doi.org/10.1016/S0929-

Bennett RS, Spurgeon DW, DeTar WR. 2011. Efficacy of four soil treatments against Fusarium oxysporum f. sp. vasinfectum race 4 on cotton. American Phytopathological Society, 95(8): 967-976. DOI: http://dx.doi.org/10.1094/PDIS-09-100696.

Bernard F. 2012. Le développement des champignons pathogènes foliaires répond à la température, mais à quelle température? Thèse de Doctorat en Agronomie. AgroParisTech, p. 145.

Bérubé ME, Vanasse A, Rioux S, Bourgeois G, Bourget N, Tremblay G, Dion Y. 2009. Effet du glyphosate et du travail du sol sur l'incidence de la fusariose de l'épi chez le blé et l'orge. Journée d'information scientifique - Grandes cultures, CRAAQ, Drummondville.

Beyer M, Roding S, Ludewig A, Verreet JA. 2004. Germination and survival of Fusarium graminearum macroconidia as affected by environmental factors. $J$. Phytopathol., 152(2): 92-97. DOI:10.1111/j.1439-0434.2003.00807.

Borras O, Santos R, Matos AP, Cabral RS, Arzola M. 2001. A first attempt to use Fusarium subglutinans culture filtrate for the selection of pineapple cultivars resistant for fusariose disease. Plant Breeding, 120(5): 435-438.

Boudoudou H, Hassikou R, Ouazzani Touhami A, Badoc A, Douria A. 2009. Paramètres physicochimiques et flore fongique des sols de rizières marocaines. Bull. Soc. Pharm. Bordeaux, 148: 17-44.

Chaudhary B, Kumar S, Lal Sharma R, Ram Jakhar S. 2018. Effect of different media, $\mathrm{pH}$ and temperature on growth and sporulation of Fusarium udun causing wilt of pigeon pea. International Journal of Current Microbiology and Applied Sciences, 6: 2005-2011.

Daami-Remadi M, Souissi A, Ben Oun H, Mansour M, Nasraoui B. 2009. Salinity effects on Fusarium wilt severity and tomato growth. Dynamic Soil, Dynamic Plant, 3(1): 61-69.

de Franqueville H, Durand GT, Cochard B, Nouy B, Breton F, Dossa J. 2011. Integrated approach for management of Fusarium disease on the oil palm. In: Proceeding of the third MPOB-IOPRI international seminar on integrated oil palm pest and diseases management. Ed. MPOB. Kuala Lumpur; 52-62.

Diabaté S, Konan KJM, Allou D, Hala N'klo F. 2013. Effet d'une souche non pathogène de Fusarium oxysporum f. sp. elaeidis sur l'expression de la fusariose chez le palmier à huile (Elaeis guineensis Jacq). Int. J. Biol. Chem. Sci., 7(3):12791288. DOI http://dx.doi.org/10.4314/ijbcs.v7i3.33.

Djébali N, Tiyab N, Gargouri S, Hessini K. 2014. Effet d'osmo-amorçage des grains sur la germination, la croissance et la résistance du blé dur à Fusarium culmorum. Journée Nationale sur la valorisation des résultats de la Recherche dans le domaine des Grandes Cultures Tunis.

Dominguez J, Negrin MA, Rodriguez CM. 2003. Evaluating soil sodium indices in soils of volcanic nature conducive or suppressive to Fusarium wilt of banana. Soil Biology \& Biochemistry, 35: 565575. DOI: $\quad 10.1016 /$ S00380717(03)00017-8.

Doohan FM, Brennan J, Cooke BM. 2003. Influence of climatic factors on Fusarium species pathogenic to cereals. European 
Journal of Plant Pathology, 109(7): 755768. DOI:10.1023/a:1026090626994.

Duffy BK, Défago G. 1999. Macro and microelements fertilizers influence the severity of Fusarium crown and root rot of tomato in a soilless production system. Horticultural Science, 34: 287291.

Ferrigo D, Raiola A, Causin R. 2016. Fusarium toxins in cereals: occurrence, legislation, factors promoting the appearance and their management. Molecules, 21(5): 627. DOI : https://doi.org/10.3390/molecules210506 27.

Ferrocino I, Chitarra W, Pugliese M, Gilardi G, Gullino ML, Garibaldi A. 2013. Effect of elevated atmospheric $\mathrm{CO} 2$ and temperature on disease severity of Fusarium oxysporum f.sp. lactucae on lettuce plants. Applied Soil Ecology, 72: 1-6.

DOI: http://dx.doi.org/10.1016/j.apsoil.2 013.05.015.

Flood J. 2006. A review of Fusarium wilt of the oil palm caused by Fusarium oxysporum f. sp. elaeidis. Phytopathology, 96(6): 660-662. DOI: 10.1094/Phyto-96-0660

Geiser DM, Lewis IML, Hakisa G, Juba JH, Miller SA. 2005. Gibberella xylarioides (anamorph: Fusarium xylariodes), a causative agent of coffee wilt disease in Africa, is a previously unrecognized member of the G. fudjikuroi species complex. Mycologia, 97: 191-201.

Gnancadja LS, Tonon DHE, Faton EMO, Douro KKO, Dannon E, Akoegninou A. 2015. Efficacité de l'agent antagoniste Trichoderma harzianum sur Fusarium oxysporum f. sp. lycopersici agent pathogène de la tomate. Int. J. Biol. Chem. Sci., 9(2): 770-782. DOI: http://dx.doi.org/10.4314/ijbcs.v9i2.17.

Gogbe DBF, Konan JN, Diabaté S, Konan EP, Koné B, Dogbo DO. 2016. Réaction phénolique de quatre clones de palmier à huile inoculés par Fusarium oxysporum f. sp. elaeidis. Int. J. Biol. Chem. Sci.,
10(2): $\quad 486-496 . \quad$ DOI: http://dx.doi.org/10.4314/ijbcs.v10i2.3.

Gordon TR. 2017. Fusarium oxysporum and the Fusarium wilt syndrome. Annual Review of Phytopathology, 55: 23-39. DOI: https://doi.org/10.1146/annurevphyto-080615-095919.

Kra KD, Diallo HA, Kobenan K, Koné D, Kouadio YJ. 2011. Diagnostic de la fusariose du bananier sur les cultivars grande naine (Musa AAA) et corne 1 (Musa $\mathrm{AAB}$ ) à la périphérie du district d'Abidjan (Côte d'ivoire). Int. J. Biol. Chem. Sci., 5(4): 1501-1514. DOI : http://dx.doi.org/10.4314/ijbcs.v5i4.16.

Lambert DH, Powelson ML, Stevenson WR. 2005. Nutritional interactions influencing diseases of potato. American Journal of Potato Research, 82(4): 309319.

Louvet J, Rouxel F, Alabouvette C. 1976. Recherches sur la résistance du sol aux maladies. I. Mise en évidence de la nature microbiologique de la résistance d'un sol au développement de la fusariose vasculaire du melon. Ann Phytopathol., 8(4): 425-436.

Keren R. 2000. Salinity. In Handbook of soil science, ed. M.E. Sumner, G3-G25. Boca Raton, FL: CRC Press.

Khilare VC, Ahmed F. 2012. Effect of different media, $\mathrm{pH}$ and temperature on the growth of Fusarium oxysporum f. sp. ciceri causing chickpea wilt. International Journal of Advanced Biological and Biomedical Research, 2(1): 99-102.

Leslie JF, Sumerell BA. 2006. The Fusarium Lab Manual. Blackwell, Ames, IA.

Ma L-J, Geiser DM, Proctor RH, Rooney AP, O'Donnell K, Trail F, Gardiner DM, Manners JM, Kazan K. 2013. Fusarium Pathogenomics. Annual Review of Microbiology, 67: 399-416. DOI: 10.1146/annurev-micro-092412-155650.

Mahdi N. 2011. Essai de lutte biologique contre la fusariose vasculaire du palmier dattier (Phoenix dactylifera). Mémoire de magister, science biologique. 
Université Mouloud Mammeri de Tizi Ouzou.

Martinez C, Michaud M, Bélanger R, Tweddell RJ. 2002. Identification of soils suppressive against Helminthosporium solani, the causal agent of potato silver scurf. Soil Biology and Biochemistry, 34: 18611868.

Mecteau MR, Arul J, Tweddell RJ. 2008. Effect of different salts on the development of Fusarium solani var coeruleum, a causal agent of potato dry rot. Phytoprotection, 89(1): 1-6. DOI : https://doi.org/10.7202/000377ar.

Mohamed L. 2006. Influence de la qualité des composts et de leurs extraits sur la protection des plantes contre les maladies fongiques. Thèse de doctorat, Université de Neuchâtel, p.161.

Nganje IB, Bangsund DA, Leistritz FL, Wilson WW, Tiapo NM. 2002. Estimating the economic impact of crop disease: the case of Fusarium head blight in U.S. Wheat and barley, National Fusarial Head Blight Forum. East Lansing Michigan State University.

Nyiransengiyumva C. 2007. Effet de différents éléments minéraux sur la croissance et le développement du champignon Helminthosporium solani, agent responsable de la gale argentée de la pomme de terre. Mémoire de maîtrise ès Sciences, Biologie Végétale. Université Laval.

Ollagnier M, Renard JL. 1976. Influence du potassium sur la résistance du palmier à huile à la Fusariose. Oléagineux, 31(5): 203-209.

Oritsejafor JJ. 1986a. Influence of moisture and $\mathrm{pH}$ on growth and survival of Fusarium oxysporum f. sp. elaeidis in soil. Transactions of the British Mycological Society, 87(4): 511-517.

Ploetz R C. 2001. Diseases of tropical crops caused by Fusarium spp. P. 295-309. In: Fusarium: Paul E. Nelson Memorial Symposium. Summerell BA, Leslie JF, Blackhouse D, Bryden WL. eds. The
American Phytopathological Society, St Paul, MN.

Ploetz R C. 2006. Fusarium wilt of banana is caused by several pathogens referred to as Fusarium oxysporum f. sp. cubense. Phytopathology, 96: 653-656. DOI: 10.1094/Phyto-96-0648.

Ramirez ML, Chulze S, Magan N. 2006. Temperature and water activity effects on growth and temporal deoxynivalenol production by two Argentinean strains of Fusarium graminearum on irradiated wheat grain. International Journal of Food Microbiology, 106(3): 291-296. DOI:10.1016/j.ijfoodmicro.2005.09.004.

Regragui A. 2005. Contribution à l'étude de l'influence de la salinité sur le couple tomate-Verticillium: conséquences physiologiques et impact sur la bioprotection des tomates contre la verticilliose. Doctorat d'État, Université Mohammed V, Faculté des Sciences, Rabat, Maroc, p. 219.

Reid TC, Hausbeck MK, Kizilkaya K. 2001. Effects of sodium chloride on commercial asparagus and of alternative forms of chloride salt on Fusarium crown and root rot. Plant Disease, 85(12): $1271-1275 . \quad$ DOI: http://dx.doi.org/10.1094/PDIS.2001.85. 12.1271.

Rossi V, Ravanetti A, Pattori E, Giosuè S. 2001. Influence of temperature and humidity on the infection of wheat spikes by some some fongi causing Fusarium head blight. Journal of Plant Pathology, 83(3): 189-198. DOI: http://dx.doi.org/10.4454/jpp.v83i3.1128

Rutherford MA. 2006. Current knowledge of coffee wilt disease, a major constraint to coffee production in Africa. Phytopathology, 96: 663-666. DOI: 10.1094/Phyto-96-0663.

Sanogo S, Yang XB. 2001. Relation of sand content, $\mathrm{pH}$ and potassium phosphorus nutrition to the development of sudden death syndrome in soybean. Canada Journal of Plant Pathology, 23: 174-180. 
DOI: 1080/070606660109506927.

Scauflaire J, Mahieu O, Louvieaux J, Foucart G, Renard F, Munaut F. 2011. Biodiversity of Fusarium species in ears and stalks of maize plants in Belgium. European Journal of Plant Pathology, 131(1): 59-66. DOI: https://doi.org/10.1007/s10658-0119787-1.

Scott JC, Gordon TR, Shaw DV, Koike ST. 2010. Effect of temperature on severity of Fusarium wilt of lettuce caused by Fusarium oxysporum f. sp. lactucae. Plant Diseases, 94(1): 13-17. DOI:10.1094/ PDIS-94-1-0013.

Shahadat HM, Ayub AM, Moni ZR, Sirajul IM, Islam MR. 2015. Effect of temperature and $\mathrm{pH}$ on the growth and sporulation of Fusarium moniliforme causing bakanae disease of rice. Scientia Agriculturae, 11(3): 151-154. DOI: 10.15192/PSCP.SA.2015.11.3.151154.

Siou D. 2013. Développement épidémique de la fusariose des épis de blé et conséquences des interactions entre espèces du complexe fusarien. Thèse de Doctorat, Université Paris-Sud 11, p.198.

Summerell AB, Laurence MH, Liew ECY, Leslie JF. 2010. Biogeography and phylogeography of Fusarium: a review. Fungal Diversity, 44: 3-13. DOI: 10.1007/s13225-010-0060-2.

Walter S, Nicholson P, Doohan FM. 2010. Research review: Action and reaction of host and pathogen during Fusarium head blight disease. New Pathologist, 185: 5466. DOI : $\quad 10.1111 /$ j. $1469-$ 8137.2009.03041.x.
Wopereis MCS, Toon DP, Idinoba SD, MarieJo D. 2008.Curriculum d'apprentissage participatif et recherche action (APRA) pour la gestion intégrée de la culture de riz de bas-fonds (GIR) en Afrique subsaharienne. Manuel technique. Le Centre du riz pour l'Afrique (ADRAO).

$\mathrm{Xu} \mathrm{X} \mathrm{M}$, Nicholson $\mathrm{P}$, Thomsett $\mathrm{M} \mathrm{A}$, Simpson D, Cooke B M, Doohan F M, Brennan J, Monaghan S, Moretti A, Mule G, Hornok L, Beki E, Tatnell J, Ritieni A, Edwards S G 2008b. Relationship between the fungal complex causing Fusarium head blight of wheat and environmental conditions. Phytopathology, 100: 763-773. DOI: 10.1094/PHYTO-98-1-0069.

$\mathrm{Xu}$ XM, Nicholson P. 2009. Community ecology of fungal pathogens causing wheat head blight. Annual Review of Phytopathology, 47: 83-103. DOI: 10.1146/annurev-phyto-080508-081737.

Zehhar G, Ouazzani TA, Badoc A, Douira A. 2006. Effet des Fusarium des eaux de rizière sur la germination et la croissance des plantules de riz. Bulletin de la société de Pharmacie de Bordeaux, 145: 7-18.

Zemmouri F, Selmaoui K, Benkirane R, Ouazzani TA, Douira A. 2015. Effet de différents sels de calcium in vitro et in vivo sur le développement des champignons de post-récolte du melon. Journal of Applied Biosciences, 93: 8748 -8759 .

DOI: http://dx.doi.org/10.4314/jab.v93i1.8. 